\title{
Position and Velocity Optimal Sensor-based Navigation Filters for UAVs
}

\author{
Pedro Batista, Carlos Silvestre, and Paulo Oliveira
}

\begin{abstract}
The paper presents the design and performance evaluation of a novel navigation solution that merges low-rate delayed GPS measurements with high-rate linear acceleration, attitude, and angular velocity measurements to estimate, in three dimensions, linear motion quantities (position, linear velocity, an acceleration of gravity) of unmanned aerial vehicles (UAVs). The design is based on the continuous-discrete Kalman filter solution for an equivalent LTI realization and allows for the natural use of frequency weights to explicitly achieve adequate disturbance rejection and measurement noise attenuation on the state estimates. The proposed solution is optimal with respect to all quantities assuming exact angular measurements and, in the presence of noisy angular quantities, it outperforms classic solutions developed in inertial coordinates. Simulation results illustrate the achievable performance in the presence of realistic measurements, including noise and delays.
\end{abstract}

\section{INTRODUCTION}

The design of Navigation and Positioning Systems plays a key role in the development of UAVs. On one hand, the acquired data sets should be properly georeferenced with respect to a given mission reference point on survey tasks. On the other hand, good navigation information is necessary for control purposes, where other quantities such as the attitude of the vehicle and the (linear and angular) velocities are also usually required. This paper presents a novel navigation solution to estimate linear motion quantities, in three dimensions, with application to UAVs.

Inertial Navigation Systems (INS) have been extensively studied in the past, as evidenced by the large number of publications on the subject, see [1], [2], [3], [4], [5], and references therein. These systems have very high short-term accuracy but, since they integrate, in open-loop, noisy quantities, the performance gets degraded over time. This limitation is successfully overcome resorting to aiding sensors such as the Global Positioning System (GPS) and several solutions have been proposed in the literature [6], [7].

The main contribution of the paper is the design and performance evaluation of a novel navigation solution that merges low-rate position GPS measurements with high-rate linear acceleration, attitude, and angular velocity measurements to provide continuous-time estimates of the linear

This work was partially supported by Fundação para a Ciência e a Tecnologia (ISR/IST plurianual funding) through the POS Conhecimento Program that includes FEDER funds and by the PTDC/EEAACR/72853/2006 OBSERVFLY Project. The work of P. Batista was supported by a PhD Student Scholarship from the POCTI Programme of FCT, SFRH/BD/24862/2005.

The authors are with the Institute for Systems and Robotics, Instituto Superior Técnico, Av. Rovisco Pais, 1049-001 Lisboa, Portugal. \{pbatista, cjs,pjcro\}eisr.ist.utl.pt motion quantities (position, velocity, and acceleration of gravity) in three dimensions. The present solution departs from previous approaches as it considers each variable in the most natural space, i.e., the space where it is measured, thus avoiding the rotation of the acceleration to inertial coordinates and the correction of the gravity and Coriolis acceleration terms. In fact, the gravity is also estimated, in body-fixed coordinates, and the Coriolis acceleration is explicitly taken into account in the model. These last two points are of major importance in the design of Navigation Systems as, due to its magnitude, any misalignment in the estimate of the acceleration of gravity or the Coriolis acceleration results in severe problems in the acceleration compensation. The advantages of the proposed solution are also evident in the case of vehicle stabilization and control. Indeed, while in classic estimation solutions the velocity of the vehicle is estimated in inertial coordinates and must be converted to body-fixed coordinates, with the proposed solution the velocity is directly estimated in body-fixed coordinates, thus reducing the impact of the noise of the attitude measurements. The same applies to the acceleration of gravity.

At the core of the proposed methodology there is a timevarying orthogonal Lyapunov transformation that renders the dynamics of the kinematic system linear time invariant (LTI). This allows for the derivation of an equivalent continuoustime Kalman filter with discrete-time delayed measurements for the LTI realization, which is then converted back to the original time-varying framework, yielding the final optimal filtering solution. Frequency weights may be included in the design to explicitly achieve adequate disturbance rejection and measurement noise attenuation on the state estimates and the proposed solution is optimal with respect to all signals assuming exact angular measurements. Interestingly enough, it is precisely in the presence of noisy angular quantities that the present solution exhibits more clearly its advantages over traditional approaches, as it will be shown. Finally, an explicit limit filtering solution is presented which does not require the solution of a Lyapunov matrix differential equation each time a new measurement arrives. This is of great practical importance since it lessens the computational cost and allows for a straightforward digital implementation of the filter.

The paper is organized as follows. The estimation problem and the system dynamics are introduced in Section II. The filter design is derived in Section III, where a limit filtering solution and alternative approaches are also discussed. 
Realistic simulation results are presented in Section IV and finally Section V summarizes the main contributions and conclusions of the paper.

Throughout the paper the symbol $\mathbf{0}_{n \times m}$ denotes an $n \times m$ matrix of zeros, $\mathbf{I}_{n}$ an identity matrix with dimension $n \times n$, and $\operatorname{diag}\left(\mathbf{A}_{1}, \ldots, \mathbf{A}_{\mathrm{n}}\right)$ a block diagonal matrix. When the dimensions are omitted the matrices are assumed of appropriate dimensions.

\section{Problem Statement}

Consider an aircraft moving with respect to an inertial reference frame $\{I\}$ and consider also a body-fixed reference frame $\{B\}$ attached to the center of mass of the vehicle. Let p denote the inertial position of the vehicle, expressed in inertial coordinates, and $\mathbf{v}$ the inertial velocity of the vehicle, expressed in body-fixed coordinates. Then, the linear motion kinematics of the vehicle are given by

$$
\dot{\mathbf{p}}(t)=\mathbf{R}(t) \mathbf{v}(t),
$$

where $\mathbf{R}$ is the rotation matrix from $\{B\}$ to $\{I\}$, that satisfies

$$
\dot{\mathbf{R}}(t)=\mathbf{R}(t) \mathbf{S}(\boldsymbol{\omega}(t)),
$$

where $\boldsymbol{\omega}$ is the angular velocity and $\mathbf{S}(\boldsymbol{\omega}(t))$ is the skewsymmetric matrix such that $\mathbf{S}(\boldsymbol{\omega}(t)) \mathbf{x}(t)=\boldsymbol{\omega}(t) \times \mathbf{x}(t)$, with $\times$ denoting the cross product between two vectors.

In addition to a GPS, which is assumed to provide the inertial position $\mathbf{p}$, the vehicle is typically equipped with an Attitude and Heading Reference System (AHRS), which provides the attitude and the angular velocity of the vehicle, and a triad of orthogonal accelerometers that provide

$$
\mathbf{a}(t)=\dot{\mathbf{v}}(t)-\mathbf{g}(t)+\mathbf{S}(\boldsymbol{\omega}(t)) \mathbf{v}(t),
$$

where $\mathrm{g}$ denotes the vector of the acceleration of gravity, expressed in body-fixed coordinates, and $\mathbf{S}(\boldsymbol{\omega}(t)) \mathbf{v}(t)$ stands for the Coriolis acceleration term. Assuming that the gravity vector is constant in inertial coordinates, it follows that its time derivative when expressed in body-fixed coordinates is simply given by

$$
\dot{\mathrm{g}}(t)=-\mathbf{S}(\boldsymbol{\omega}(t)) \mathbf{g}(t) .
$$

The dynamics of the linear motion quantities can thus be written as

$$
\left\{\begin{array}{l}
\dot{\mathbf{p}}(t)=\mathbf{R}(t) \mathbf{v}(t) \\
\dot{\mathbf{v}}(t)=\mathbf{u}(t)+\mathbf{g}(t)-\mathbf{S}(\boldsymbol{\omega}(t)) \mathbf{v}(t) \\
\dot{\mathbf{g}}(t)=-\mathbf{S}(\boldsymbol{\omega}(t)) \mathbf{g}(t) \\
\boldsymbol{\psi}(t)=\mathbf{p}(t)
\end{array}\right.
$$

where $\boldsymbol{\psi}$ denotes the system output and $\mathbf{u}=\mathbf{a}$ is the system input. Although these equations are standard, classic navigation approaches do not use this form. Instead, the acceleration is converted to inertial coordinates and the gravity and Coriolis terms canceled. The novelty of the solution proposed in the paper steams from expressing each quantity in the sensor space, i.e., in the same reference frame of the sensor, thus reducing the impact of the noise of the attitude measurements. Moreover, while in inertialbased solutions the angular velocity measurement is quite important to the estimation of angular quantities but somehow neglected in the estimation of the linear quantities, that does not happen using this framework since it appears explicitly in the dynamics of the system.

The estimation of all quantities should take place in a continuous-time framework, avoiding the undesirable approximation of the system dynamics and facilitating the project of the filters. Moreover, in the case of UAVs, where controllers usually require the vehicle state to actuate the control surfaces and thrusters, this is of particular importance. Sensors where high sampling rates are available are not a problem as there exist simple and effective numerical algorithms that solve the integration problem, but the GPS offers only low sampling rate updates. What is more, it does so with time-varying delays that must be taken into account in the design, particularly for high-speed vehicles such as UAVs. Suppose that the output of the system is sampled at a constant rate and that the measurements arrive with timevarying delays, as given by

$$
\boldsymbol{\psi}_{k}^{d}=\boldsymbol{\psi}^{d}\left(t_{k}^{d}\right)=\boldsymbol{\psi}\left(t_{k}\right)+\mathbf{n}_{d}\left(t_{k}\right), k \in \mathbb{N},
$$

where $\mathbf{n}_{d}$ is discrete-time zero-mean white noise with covariance $N_{d} \mathbf{I}, t_{k}^{d}$ denotes the instant of arrival of the measurement $k$, and $t_{k}$ denotes the sampling instant of measurement $k$, given by

$$
t_{k}=k T, k \in \mathbb{N},
$$

where $T$ denotes the sampling period. A temporal diagram that illustrates the output sampling is depicted in Fig. 1.

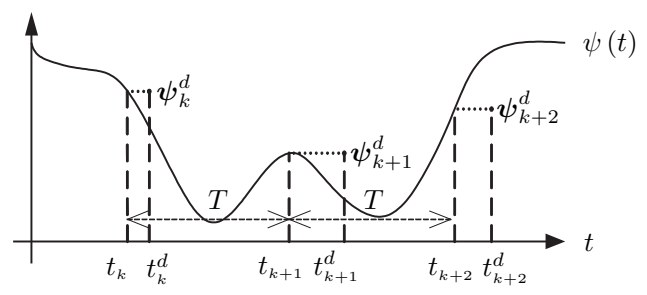

Fig. 1. Temporal Diagram of GPS sampling

To complete the formulation of the system dynamics, it is interesting to add continuous-time system disturbances. To that purpose, consider the system dynamics

$$
\left\{\begin{array}{l}
\dot{\boldsymbol{\eta}}_{p}(t)=\boldsymbol{A}_{p}(t) \boldsymbol{\eta}_{p}(t)+\mathbf{B}_{p} \mathbf{u}(t)+\boldsymbol{d}(t) \\
\boldsymbol{\psi}(t)=\mathbf{C}_{p} \boldsymbol{\eta}_{p}(t)+\boldsymbol{n}(t)
\end{array},\right.
$$

where $\boldsymbol{\eta}_{p}(t)=\left[\mathbf{p}^{T}(t) \boldsymbol{v}^{T}(t) \mathbf{g}^{T}(t)\right]^{T} \in \mathbb{R}^{9}$ is the system state, $\boldsymbol{d}(t)$ denotes the system disturbances input, $\boldsymbol{n}(t)$ denotes a disturbance that affects the output of the system,

$$
\mathcal{A}_{p}(t)=\left[\begin{array}{ccc}
\mathbf{0} & \mathbf{R}(t) & \mathbf{0} \\
\mathbf{0} & -\mathbf{S}(\boldsymbol{\omega}(t)) & \mathbf{I} \\
\mathbf{0} & \mathbf{0} & -\mathbf{S}(\boldsymbol{\omega}(t))
\end{array}\right], \mathbf{B}_{p}=\left[\begin{array}{l}
\mathbf{0} \\
\mathbf{I} \\
\mathbf{0}
\end{array}\right]
$$

and $\mathbf{C}_{p}=\left[\begin{array}{lll}\mathbf{I}_{3} & \mathbf{0} & \mathbf{0}\end{array}\right]$. For design purposes consider that the disturbance input $\boldsymbol{d}(t)$ is given by

$$
\boldsymbol{d}(\boldsymbol{t}):=\mathbf{T}^{T}(t) \mathbf{d}(t),
$$


where

$$
\mathbf{T}(t):=\operatorname{diag}(\mathbf{I}, \mathbf{R}(\mathrm{t}), \mathbf{R}(\mathrm{t}))
$$

and $\mathbf{d}(t)$ is the output of a stable LTI system $\mathcal{W}_{d}$ driven by zero-mean, unit intensity white noise. Similarly, suppose that $\boldsymbol{n}(t)$ is the output of a stable LTI system $\mathcal{W}_{n}$, also driven by zero-mean, unit intensity white noise. Further assume that $\mathcal{W}_{n}$ is a stricly proper system. If that was not the case, $\boldsymbol{\psi}(t)$ would contain white noise components that would lead to a discrete sequence of measurements with infinite variance. This is, nevertheless, a mild assumption since in nature there are no processes with infinite energy. Let $\mathbf{x}_{d}(t)$ and $\mathbf{x}_{n}(t)$ denote the internal states of state space realizations $\left(\mathbf{A}_{d}, \mathbf{B}_{d}, \mathbf{C}_{d}, \mathbf{D}_{d}\right)$ and $\left(\mathbf{A}_{n}, \mathbf{B}_{n}, \mathbf{C}_{n}, \mathbf{0}\right)$ of $\mathcal{W}_{d}$ and $\mathcal{W}_{n}$, respectively. Then, the augmented system dynamics can be written as

$$
\left\{\begin{array}{l}
\dot{\boldsymbol{\eta}}(t)=\mathcal{A}(t) \boldsymbol{\eta}(t)+\mathcal{B}_{p} \mathbf{u}(t)+\mathcal{B}(t) \mathbf{w}(t) \\
\boldsymbol{\psi}_{k}^{d}=\mathcal{C} \boldsymbol{\eta}\left(t_{k}\right)+\mathbf{n}_{d}\left(t_{k}\right), k \in \mathbb{N}
\end{array}\right.
$$

where $\boldsymbol{\eta}(t):=\left[\boldsymbol{\eta}_{p}^{T}(t) \mathbf{x}_{d}^{T} \mathbf{x}_{n}^{T}\right]^{T}$

$$
\begin{gathered}
\mathcal{A}(t)=\left[\begin{array}{ccc}
\mathcal{A}_{p}(t) & \mathbf{T}^{T}(t) \mathbf{C}_{d} & \mathbf{0} \\
\mathbf{0} & \mathbf{A}_{d} & \mathbf{0} \\
\mathbf{0} & \mathbf{0} & \mathbf{A}_{n}
\end{array}\right], \\
\mathcal{B}_{p}=\left[\begin{array}{c}
\mathbf{B}_{p} \\
\mathbf{0} \\
\mathbf{0}
\end{array}\right], \quad \mathcal{B}(t)=\left[\begin{array}{cc}
\mathbf{T}^{T}(t) \mathbf{D}_{d} & \mathbf{0} \\
\mathbf{B}_{d} & \mathbf{0} \\
\mathbf{0} & \mathbf{B}_{n}
\end{array}\right],
\end{gathered}
$$

and $\mathcal{C}=\left[\mathbf{C}_{p}|\mathbf{0}| \mathbf{C}_{n}\right]$. The overall design setup is depicted in Fig. 2, where $\mathbf{w}=\left[\mathbf{w}_{d}^{T} \mathbf{w}_{n}^{T}\right]^{T}$ is zero-mean, unit intensity white noise. The system disturbance model $\mathcal{W}_{d}$ and the output disturbance model $\mathcal{W}_{n}$ may be useful, e.g., to model colored noise of the accelerometer and the GPS, respectively. Also, notice that the transformation matrix $\mathbf{T}(t)$ preserves the norm of the disturbances that affect the states $\mathbf{p}, \mathbf{v}$, and $\mathrm{g}$, and the directionally is only affected for the disturbances on the latter two, $\mathbf{v}$ and $\mathbf{g}$.

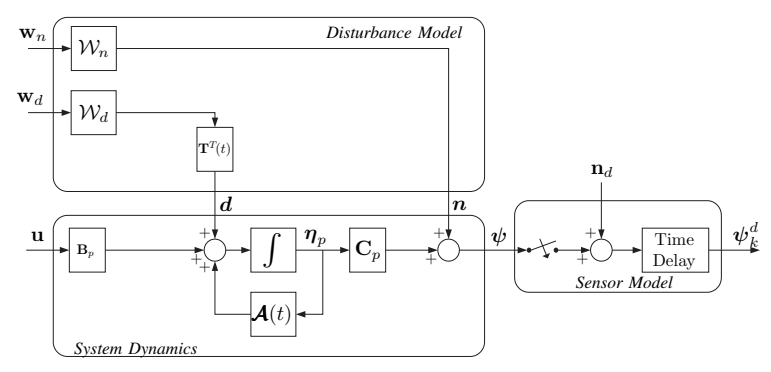

Fig. 2. Filter design setup

The problem considered in the paper is the design of an optimal continuous-time filter with discrete-time delayed measurements for the linear time-varying system (1).

\section{FILTER DESIGN}

This section presents the filter design concepts that are at the core of the navigation solution proposed in the paper. The derivation of the filter equations is detailed in
Section III-A, whereas Section III-B suggests a time-varying filtering limit solution. Implementation issues and alternative filtering solutions for the problems addressed in the paper are discussed in Section III-C.

\section{A. Filter Equations}

In order to derive the optimal Kalman filter, consider the Lyapunov coordinate transformation similar to the one first proposed in [8] and define

$$
\mathbf{x}(t):=\mathbf{T}_{c}(t) \boldsymbol{\eta}(t)
$$

where

$$
\mathbf{T}_{c}(t):=\operatorname{diag}(\mathbf{T}(\mathrm{t}), \mathbf{I}, \mathbf{I}) .
$$

Then, the dynamics of the system expressed in this new coordinate space can be written as

$$
\left\{\begin{array}{l}
\dot{\mathbf{x}}(t)=\mathbf{A x}(t)+\mathbf{B w}(t)+\mathbf{T}_{c}(t) \mathcal{B}_{p} \mathbf{u}(t) \\
\mathbf{y}_{k}^{d}=\mathbf{C x}\left(t_{k}\right)+\mathbf{n}_{d}\left(t_{k}\right), k \in \mathbb{N}
\end{array}\right.
$$

where

$$
\begin{gathered}
\mathbf{A}:=\left[\begin{array}{ccc}
\mathbf{A}_{p} & \mathbf{C}_{d} & \mathbf{0} \\
\mathbf{0} & \mathbf{A}_{d} & \mathbf{0} \\
\mathbf{0} & \mathbf{0} & \mathbf{A}_{n}
\end{array}\right], \mathbf{A}_{p}:=\left[\begin{array}{lll}
\mathbf{0} & \mathbf{I} & \mathbf{0} \\
\mathbf{0} & \mathbf{0} & \mathbf{I} \\
\mathbf{0} & \mathbf{0} & \mathbf{0}
\end{array}\right], \\
\mathbf{B}:=\left[\begin{array}{cc}
\mathbf{D}_{d} & 0 \\
\mathbf{B}_{d} & \mathbf{0} \\
\mathbf{0} & \mathbf{B}_{n}
\end{array}\right],
\end{gathered}
$$

and $\mathbf{C}:=\mathcal{C T}_{c}^{T}\left(t_{k}\right)=\left[\mathbf{C}_{p}|\mathbf{0}| \mathbf{C}_{n}\right]=\mathcal{C}$. The advantage of using this coordinate transformation is that the state dynamics become linear time invariant in what concerns the dependence on the state and the noise. The deterministic input is simply replicated in the filter design.

The Kalman filter for the continuous-discrete system (3) is given by

$$
\left\{\begin{array}{l}
\dot{\hat{\mathbf{x}}}(t)=\mathbf{A} \hat{\mathbf{x}}(t)+\mathbf{T}_{c}(t) \mathcal{B}_{p} \mathbf{u}(t), t_{k}^{d} \leq t<t_{k+1}^{d} \\
\hat{\mathbf{x}}\left(t_{k}^{d}\right)=\hat{\mathbf{x}}\left(t_{k}^{d^{-}}\right)+\mathbf{\Phi}\left(t_{k}^{d}, t_{k}\right) \mathbf{K}\left(t_{k}\right)\left[\mathbf{y}\left(t_{k}^{d}\right)-\mathbf{C} \hat{\mathbf{x}}\left(t_{k}\right)\right] \\
\hat{\mathbf{x}}\left(t_{0}\right)=\hat{\mathbf{x}}_{0}=\mathbf{T}_{c}\left(t_{0}\right) \hat{\boldsymbol{\eta}}_{0}
\end{array}\right.
$$

where $t_{0}^{d}=t_{0}, \hat{\boldsymbol{\eta}}_{0}$ is the initial state estimate, $\boldsymbol{\Phi}\left(t_{k}^{d}, t_{k}\right)$ is the transition matrix

$$
\boldsymbol{\Phi}\left(t_{k}^{d}, t_{k}\right)=e^{\mathbf{A}\left(t_{k}^{d}-t_{k}\right)},
$$

that simply propagates the discrete update to compensate for the measurement delays, and $\mathbf{K}\left(t_{k}\right)$ is the Kalman matrix gain, given by

$$
\mathbf{K}\left(t_{k}\right)=\frac{1}{N_{d}} \mathbf{P}_{c}\left(t_{k}\right) \mathbf{C}^{T}
$$

where $\mathbf{P}_{c}(t)$ denotes the covariance of the error of the estimate of the state at time $t$ assuming there are no measurement delays, whose dynamics are given by

$$
\left\{\begin{array}{l}
\dot{\mathbf{P}}_{c}(t)=\mathbf{A P}_{c}(t)+\mathbf{P}_{c}(t) \mathbf{A}^{T}+\mathbf{B B}^{T}, t_{k-1} \leq t<t_{k}, k \in \mathbb{N} \\
\mathbf{P}_{c}\left(t_{0}\right)=\mathbf{P}_{c 0}=\mathbf{T}_{c}\left(t_{0}\right) \boldsymbol{P}_{c 0} \mathbf{T}_{c}^{T}\left(t_{0}\right)
\end{array},\right.
$$

where $\mathcal{P}_{c 0}=\mathcal{P}_{0} \succ \mathbf{0}$ is the initial covariance matrix and the discrete update at $t_{k}$ is given by

$$
\begin{aligned}
\mathbf{P}_{c}\left(t_{k}\right)= & \mathbf{P}_{c}\left(t_{k}{ }^{-}\right)-\mathbf{P}_{c}\left(t_{k}{ }^{-}\right) \mathbf{C}^{T} \\
& {\left[\mathbf{C P}_{c}\left(t_{k}{ }^{-}\right) \mathbf{C}^{T}+N_{d} \mathbf{I}\right]^{-1} \mathbf{C P}_{c}\left(t_{k}{ }^{-}\right) . }
\end{aligned}
$$


The filter equations in the original coordinate space can be recovered by inverting the coordinate transformation (2), which gives

$$
\left\{\begin{array}{l}
\dot{\hat{\boldsymbol{\eta}}}(t)=\boldsymbol{\mathcal { A }}(t) \hat{\boldsymbol{\eta}}(t)+\mathcal{B}_{p} \mathbf{u}(t), t_{k-1}^{d} \leq t<t_{k}^{d}, k \in \mathbb{N} \\
\hat{\boldsymbol{\eta}}\left(t_{k}^{d}\right)=\hat{\boldsymbol{\eta}}\left(t_{k}^{d^{-}}\right)+\boldsymbol{\Phi}\left(t_{k}^{d}, t_{k}\right) \mathcal{K}\left(t_{k}\right)\left[\boldsymbol{\psi}_{k}^{d}-\mathcal{C} \hat{\boldsymbol{\eta}}\left(t_{k}\right)\right] \\
\hat{\boldsymbol{\eta}}\left(t_{0}\right)=\hat{\boldsymbol{\eta}}_{0}
\end{array}\right.
$$

where $\boldsymbol{\Phi}\left(t_{k}^{d}, t_{k}\right)$ denotes the transition matrix associated with $\mathcal{A}(t)$, simply written as

$$
\boldsymbol{\Phi}\left(t_{k}^{d}, t_{k}\right)=\mathbf{T}_{c}^{T}\left(t_{k}^{d}\right) \boldsymbol{\Phi}\left(t_{k}^{d}, t_{k}\right) \mathbf{T}_{c}\left(t_{k}\right),
$$

and $\mathcal{K}\left(t_{k}\right)$ denotes the Kalman matrix gain, given by

$$
\mathcal{K}\left(t_{k}\right)=\frac{1}{N_{d}} \mathcal{P}_{c}\left(t_{k}\right) \mathcal{C}^{T},
$$

where $\mathcal{P}_{c}(t)$ denotes the covariance matrix of the error of the estimate of $\boldsymbol{\eta}$ at time $t$ if there were no measurement delays, which can be expressed as

$$
\mathcal{P}_{c}(t)=\mathbf{T}_{c}^{T}(t) \mathbf{P}_{c}(t) \mathbf{T}_{c}(t)
$$

The following theorem summarizes the main result of this section.

Theorem 1: Consider the generalized system dynamics as depicted in Fig. 2, where $\mathbf{w}=\left[\mathbf{w}_{d}^{T} \mathbf{w}_{n}^{T}\right]^{T}$ is zeromean, unit intensity, continuous-time white noise and $\mathbf{n}_{d}$ is zero-mean, discrete-time white noise, with covariance matrix $N_{d} \mathbf{I}$. Assume that $\boldsymbol{\eta}\left(t_{0}\right), \mathbf{w}(t)$, and $\mathbf{n}_{d}\left(t_{k}\right)$ are mutually uncorrelated for all time. Let $t_{0}^{d}=t_{0}, \hat{\boldsymbol{\eta}}_{0}$ be the initial state estimate, and $\mathcal{P}_{0}$ the initial error covariance matrix. Then, the optimal Kalman filter is given by (5) and the error covariance matrix $\mathcal{P}(t)$ is given by

$$
\mathcal{P}(t)=\mathbf{T}_{c}^{T}(t) \mathbf{P}(t) \mathbf{T}_{c}(t)
$$

where $\mathbf{P}(t)$ satisfies

$$
\left\{\begin{array}{l}
\dot{\mathbf{P}}(t)=\mathbf{A} \mathbf{P}(t)+\mathbf{P}(t) \mathbf{A}^{T}+\mathbf{B B}^{T}, t_{k-1}^{d} \leq t<t_{k}^{d}, k \in \mathbb{N} \\
\mathbf{P}\left(t_{0}^{d}\right)=\mathbf{P}_{0}
\end{array}\right.
$$

and the discrete update is given by the propagation of $\mathbf{P}_{c}\left(t_{k}\right)$, which can be written as

$$
\begin{aligned}
\mathbf{P}\left(t_{k}^{d}\right)= & \mathbf{P}\left(t_{k}^{d^{-}}\right)-\mathbf{\Phi}\left(t_{k}^{d}, t_{k}\right) \mathbf{P}\left(t_{k}\right) \mathbf{C}^{T} \\
& {\left[\mathbf{C P}\left(t_{k}\right) \mathbf{C}^{T}+\mathbf{I}\right]^{-1} \mathbf{C} \mathbf{P}\left(t_{k}\right) \boldsymbol{\Phi}^{T}\left(t_{k}^{d}, t_{k}\right), k \in \mathbb{N} . }
\end{aligned}
$$

Proof: The Kalman filter dynamics (5) are standard, where $\boldsymbol{\Phi}\left(t_{k}^{d}, t_{k}\right)$ accounts for the correction due to the measurement delays. To show that the Kalman gain is given by (6) it remains to show that (7) is the solution of

$$
\dot{\mathcal{P}}_{c}(t)=\mathcal{A}(t) \mathcal{P}_{c}(t)+\mathcal{P}_{c}(t) \mathcal{A}^{T}(t)+\mathcal{B}(t) \mathcal{B}^{T}(t), t_{k-1} \leq t<t_{k}, k \in \mathbb{N},
$$

with $\mathcal{P}_{c}\left(t_{0}\right)=\mathcal{P}_{c 0}$ and

$$
\begin{aligned}
\mathcal{P}_{c}\left(t_{k}\right) & =\mathcal{P}_{c}\left(t_{k}{ }^{-}\right)-\mathcal{P}_{c}\left(t_{k}{ }^{-}\right) \mathcal{C}^{T} \\
& {\left[\mathcal{C P}_{c}\left(t_{k}{ }^{-}\right) \mathcal{C}^{T}+N_{d} \mathbf{I}\right]^{-1} \mathcal{C} \mathcal{P}_{c}\left(t_{k}{ }^{-}\right) . }
\end{aligned}
$$

Substituting (4) in (7) immediately yields (11). After a few algebraic manipulations, the time derivative of (7) gives (10). The actual error covariance matrix does not coincide with $\mathcal{P}_{c}(t)$ due to the measurement delays. Thus, between arrivals of the measurements, the covariance matrix is integrated in open-loop as given by (8). At the time of arrival $t_{k}^{d}$, the covariance is updated so that it coincides with $\mathcal{P}_{c}\left(t_{k}^{d}\right)$, i.e., $\mathcal{P}\left(t_{k}^{d}\right)=\mathcal{P}_{c}\left(t_{k}^{d}\right)$ or, using (7),

$$
\mathcal{P}\left(t_{k}^{d}\right)=\mathbf{T}_{c}^{T}\left(t_{k}^{d}\right) \mathbf{P}_{c}\left(t_{k}^{d}\right) \mathbf{T}_{c}\left(t_{k}^{d}\right) .
$$

The covariance matrix $\mathbf{P}_{c}\left(t_{k}^{d}\right)$ can be written as

$$
\begin{aligned}
\mathbf{P}_{c}\left(t_{k}^{d}\right) & =\boldsymbol{\Phi}\left(t_{k}^{d}, t_{k}\right) \mathbf{P}_{c}\left(t_{k}\right) \boldsymbol{\Phi}^{T}\left(t_{k}^{d}, t_{k}\right) \\
& +\int_{t_{k}}^{t_{k}^{d}} \boldsymbol{\Phi}\left(t_{k}^{d}, \tau\right) \boldsymbol{B}(\tau) \boldsymbol{B}^{T}(\tau) \boldsymbol{\Phi}^{T}\left(t_{k}^{d}, \tau\right) d \tau .
\end{aligned}
$$

Substituting (4) in (13), and since $\mathbf{P}_{c}\left(t_{k}{ }^{-}\right)=\mathbf{P}\left(t_{k}\right)$, gives

$$
\begin{aligned}
\mathbf{P}_{c}\left(t_{k}^{d}\right)= & \boldsymbol{\Phi}\left(t_{k}^{d}, t_{k}\right) \mathbf{P}\left(t_{k}\right) \boldsymbol{\Phi}^{T}\left(t_{k}^{d}, t_{k}\right) \\
& +\int_{t_{k}}^{t_{k}^{d}} \boldsymbol{\Phi}\left(t_{k}^{d}, \tau\right) \boldsymbol{B}(\tau) \boldsymbol{B}^{T}(\tau) \boldsymbol{\Phi}^{T}\left(t_{k}^{d}, \tau\right) d \tau \\
- & \boldsymbol{\Phi}\left(t_{k}^{d}, t_{k}\right) \mathbf{P}\left(t_{k}\right) \mathbf{C}^{T}\left[\mathbf{C P}\left(t_{k}\right) \mathbf{C}^{T}+N_{d} \mathbf{I}\right]^{-1} \\
& \mathbf{C P}\left(t_{k}\right) \boldsymbol{\Phi}^{T}\left(t_{k}^{d}, t_{k}\right) .
\end{aligned}
$$

Now, notice that

$$
\begin{aligned}
\mathbf{P}\left(t_{k}^{d^{-}}\right) & =\boldsymbol{\Phi}\left(t_{k}^{d}, t_{k}\right) \mathbf{P}\left(t_{k}\right) \boldsymbol{\Phi}^{T}\left(t_{k}^{d}, t_{k}\right) \\
& +\int_{t_{k}}^{t_{k}^{d}} \boldsymbol{\Phi}\left(t_{k}^{d}, \tau\right) \boldsymbol{B}(\tau) \boldsymbol{B}^{T}(\tau) \boldsymbol{\Phi}^{T}\left(t_{k}^{d}, \tau\right) d \tau .
\end{aligned}
$$

Thus, (14) may be rewritten as

$$
\begin{aligned}
\mathbf{P}_{c}\left(t_{k}^{d}\right)= & \mathbf{P}\left(t_{k}^{d^{-}}\right) \\
- & \boldsymbol{\Phi}\left(t_{k}^{d}, t_{k}\right) \mathbf{P}\left(t_{k}\right) \mathbf{C}^{T}\left[\mathbf{C P}\left(t_{k}\right) \mathbf{C}^{T}+N_{d} \mathbf{I}\right]^{-1} \\
& \mathbf{C P}\left(t_{k}\right) \boldsymbol{\Phi}^{T}\left(t_{k}^{d}, t_{k}\right) .
\end{aligned}
$$

But (15) corresponds to (9), which means, from (12), that

$$
\mathcal{P}\left(t_{k}^{d}\right)=\mathbf{T}_{c}^{T}\left(t_{k}^{d}\right) \mathbf{P}\left(t_{k}^{d}\right) \mathbf{T}_{c}\left(t_{k}^{d}\right),
$$

which concludes the proof.

\section{B. Filtering Limit Solution}

In the previous section the filter equations were derived by means of an appropriate Lyapunov transformation that rendered the dynamics linear time invariant, as given by (3). Under appropriate stabilizability and detectability hypothesis, it is well known that the Kalman filter for the continuousdiscrete LTI system (3) converges to an asymptotically stable steady-state solution. The existence of a special relationship between (3) and (1) induces a limit filtering solution for the the system at hand.

Define

$$
\begin{gathered}
\overline{\boldsymbol{\Phi}}:=\boldsymbol{\Phi}(T, 0), \\
\mathbf{Q}:=\int_{0}^{T} \boldsymbol{\Phi}(T, \tau) \mathbf{B B}^{T} \boldsymbol{\Phi}^{T}(T, \tau) d \tau,
\end{gathered}
$$

and suppose that the pairs $(\overline{\mathbf{\Phi}}, \mathbf{Q})$ and $(\overline{\mathbf{\Phi}}, \mathbf{C})$ are stabilizable and detectable, respectively. Notice that this only depends on the choice of the filters $\mathcal{W}_{d}$ and $\mathcal{W}_{n}$. Under these 
conditions the estimation error covariance matrix $\mathbf{P}_{c}\left(t_{k}\right)$ converges to

$$
\lim _{k \rightarrow \infty} \mathbf{P}_{c}\left(t_{k}\right)=\mathbf{P}_{c}^{\infty},
$$

with

$$
\mathbf{P}_{c}^{\infty}:=\mathbf{P}_{c}^{r}-\mathbf{P}_{c}^{r} \mathbf{C}^{T}\left(\mathbf{C P}_{c}^{r} \mathbf{C}^{T}+N_{d} \mathbf{I}\right)^{-1} \mathbf{C P}_{c}^{r},
$$

where $\mathbf{P}_{c}^{r}$ is the solution of the discrete-time algebraic Riccati equation

$$
\mathbf{P}_{c}^{r}=\overline{\boldsymbol{\Phi}} \mathbf{P}_{c}^{r} \overline{\boldsymbol{\Phi}}^{T}+\mathbf{Q}-\overline{\boldsymbol{\Phi}} \mathbf{P}_{c}^{r} \mathbf{C}^{T}\left[\mathbf{C} \mathbf{P}_{c}^{r} \mathbf{C}+N_{d} \mathbf{I}\right]^{-1} \mathbf{C} \mathbf{P}_{c}^{r} \overline{\boldsymbol{\Phi}}^{T} .
$$

From (6), (7), and (16) it follows that the Kalman gain converges to the limit solution

$$
\lim _{k \rightarrow \infty} \mathcal{K}\left(t_{k}\right)=\frac{1}{N_{d}} \mathbf{T}_{c}^{T}\left(t_{k}\right) \mathbf{P}_{c}^{\infty} \mathbf{T}_{c}\left(t_{k}\right) \mathcal{C}^{T} .
$$

The Kalman filter that results from using the limit solution for the gain (17) is still time-varying due to the inherent nature of the system dynamics. However, the gain is now readily obtained and does not require the online solution of the Lyapunov matrix differential equation (8), which could be computationally expensive.

\section{Alternative Realizations and Implementation}

It was assumed throughout all the derivations that the output of the system was sampled at a constant rate. This constraint may be discarded and the previous filter modified accordingly to allow aperiodic sampling of the system output. However, in that situation, a limit filtering solution, as reported in Section III-B, does not exist. In fact, for aperiodic sampling, a Lyapunov matrix differential equation must be solved each time a new measurement arrives in order to compute the optimal gain, which is computationally expensive.

In the paper it was chosen a Kalman filtering setup, assuming zero-mean white noise and zero-mean white disturbances. However, it should be said that the optimal $\mathcal{H}_{\infty}$ filter for the continuous-time system with discrete-time delayed measurements presented in Section II may be derived following similar steps, see [9] for details on the general $\mathcal{H}_{\infty}$ filtering setup for sampled-data systems.

Figure 3 presents the block diagram implementation of the proposed solution, where the dashed lines represent injection of initial conditions on the various blocks each time a new measurement is available. Although the system dynamics presented in the paper are continuous, it turns out, in practice, that the filter is implemented in a discrete-time framework. Thus, the open-loop propagation of the system state estimate is done resorting to integration algorithms, namely, numerical algorithms such as Runge-Kutta methods. The signals $\boldsymbol{\omega}, \mathbf{R}$, and $\mathbf{u}$ are thus sampled at high rates in order to allow precise numerical integration of the state estimate.

\section{Simulation Results}

In order to evaluate the performance achieved with the proposed navigation solution, simulations were carried out using a full nonlinear model (see [10] for further details) of a small scale airplane. The simulations are carried out in

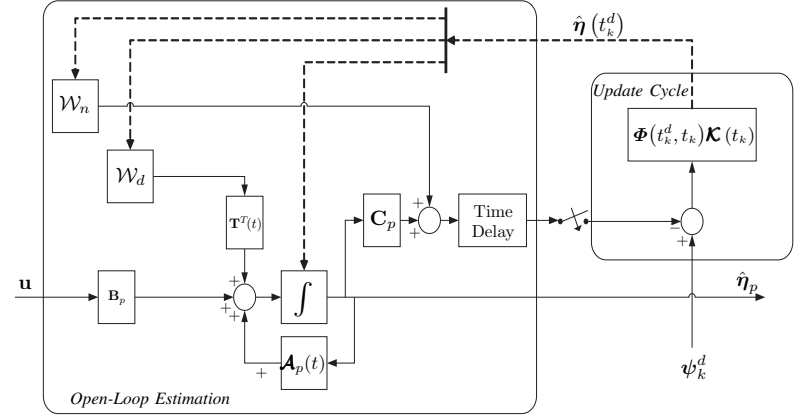

Fig. 3. Block Diagram of the Filter Implementation

the presence of wind disturbances and the path-following controller proposed in [10] is employed to generate the trajectory depicted in Fig. 4.

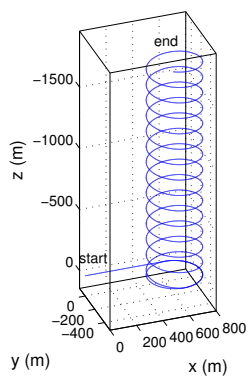

Fig. 4. Trajectory described by the UAV

In the simulation the GPS measurements are corrupted with additive zero-mean white noise, with standard deviation of $1 \mathrm{~m}$, and arrive with delays that follow an exponential distribution with parameter $\lambda=0.075$, whereas the sampling rate is $1 \mathrm{~Hz}$. The remaining sensors, viz. the AHRS and the triad of accelerometers, are sampled at $100 \mathrm{~Hz}$. In addition, the acceleration and the angular velocity measurements are corrupted by additive zero-mean Gaussian noises, with standard deviations of $0.006 \mathrm{~m} / \mathrm{s}^{2}$ and $0.02 \%$, respectively, and the attitude of the vehicle, assumed to be given by the roll, pitch, and yaw Euler angles, corrupted by additive zero-mean white Gaussian noises with standard deviation of $0.2^{\circ}$ for the roll and pitch and $1^{\circ}$ for the yaw. The open-loop propagation of the system state estimates was carried out with a RungeKutta method of fourth-order. In order to properly tune the behavior of the filter the frequency weight transfer functions were chosen as $\mathbf{W}_{d}=0.001 \mathbf{I}_{6}, \mathbf{W}_{n}=\mathbf{0}$, and $N_{d}=1$.

The evolution of the filter error variables is shown in Fig. 5. The initial transients arise due to the mismatch of the initial conditions of the states of the filter and can be considered as a warming up time of 3 minutes of the corresponding Integrated Navigation System. Notice that this can be drastically reduced by proper initialization of the filter variables. The filter error variables are shown in greater detail in Fig. 6. From the various plots it can be concluded that the measurement noise is highly attenuated by the filter, producing very accurate estimates of the inertial position of 
the vehicle, velocity, and acceleration of gravity. The delays in the position measurements are also dealt with successfully.
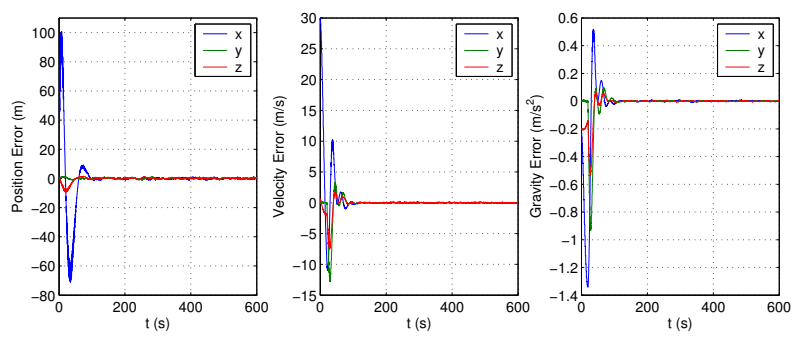

Fig. 5. Time evolution of the Kalman filter error variables
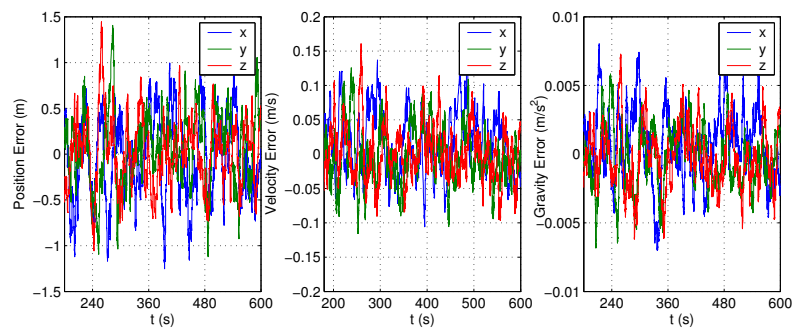

Fig. 6. Detailed evolution of the Kalman filter error variables

To compare the strategy proposed in the paper with classic strategies, the previous simulation was repeated with a Kalman filter completely developed in inertial coordinates. The evolution of the resulting filter error is shown in Fig. 7 and, in greater detail, in Fig. 8. The differences between the
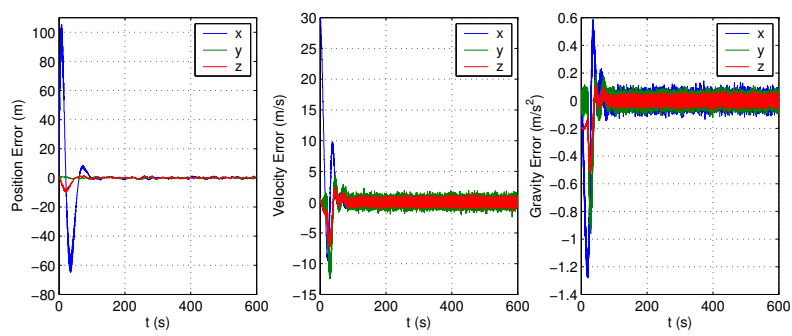

Fig. 7. Time evolution of the inertial Kalman filter error variables
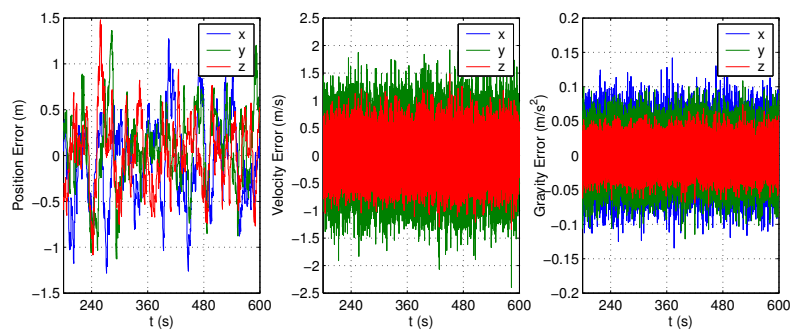

Fig. 8. Detailed evolution of the inertial Kalman filter error variables

proposed solution and the classic solution are highlighted in Table I, where the steady-state standard deviations of the errors for both strategies are presented. The proposed sensor-based strategy clearly outperforms the classic inertial solution, especially in what concerns the estimation of the velocity and acceleration of gravity. The position is not as affected as it is estimated in inertial coordinates in both solutions.

\begin{tabular}{c|c|c}
\hline Standard deviation of the errors & Proposed Filter & Inertial Filter \\
\hline Position $-x$ axis $(\mathrm{m})$ & 0.4796 & 0.4992 \\
Position $-y$ axis $(\mathrm{m})$ & 0.3669 & 0.3645 \\
Position $-z$ axis $(\mathrm{m})$ & 0.3225 & 0.3186 \\
Velocity $-x$ axis $(\mathrm{m} / \mathrm{s})$ & 0.0454 & 0.0519 \\
Velocity $-y$ axis $(\mathrm{m} / \mathrm{s})$ & 0.0357 & 0.5229 \\
Velocity $-z$ axis $(\mathrm{m} / \mathrm{s})$ & 0.0356 & 0.3413 \\
Gravity $-x$ axis $\left(\mathrm{m} / \mathrm{s}^{2}\right)$ & 0.0023 & 0.0342 \\
Gravity $-y$ axis $\left(\mathrm{m} / \mathrm{s}^{2}\right)$ & 0.0018 & 0.0292 \\
Gravity $-z$ axis $\left(\mathrm{m} / \mathrm{s}^{2}\right)$ & 0.0020 & 0.0184
\end{tabular}

STANDARD DEVIATION OF THE FILTER ERRORS

\section{CONCLUSIONS}

The paper presented the design and performance evaluation of a novel sensor-based navigation solution that merges low-rate delayed GPS measurements with high-rate linear acceleration, attitude, and angular velocity measurements to estimate, in three dimensions, linear motion quantities (position, linear velocity, an acceleration of gravity) of unmanned aerial vehicles (UAVs). The design is based on the continuous-discrete Kalman filter solution for an equivalent LTI realization and allows for the natural use of frequency weights to explicitly achieve adequate disturbance rejection and measurement noise attenuation on the state estimates. The proposed solution is optimal with respect to all quantities assuming exact angular measurements and, in the presence of noisy angular quantities, it outperforms classic solutions developed in inertial coordinates. Simulation results were discussed that illustrate the achievable performance in the presence of realistic measurements, including noise and delays.

\section{REFERENCES}

[1] K. R. Britting, Inertial Navigation Systems Analysis. John Wiley \& Sons Inc, 1972.

[2] A. B. Chatfield, Fundamentals of High Accuracy Inertial Navigation. AIAA, 1997.

[3] A. King, "Inertial Navigation Forty Years of Evolution," GEC Review, vol. 13, no. 3, pp. 140-149, 1998.

[4] S. Merhav, Aerospace Sensor Systems and Applications. Springer, 1998.

[5] M. Kayton and W. Fried, Avionics Navigation Systems. WileyInterscience, 1997.

[6] R. Brown and P. Hwang, Introduction to Random Signals and Applied Kalman Filtering (3rd ed.). John Wiley \& Sons, Inc, 1997.

[7] Y. Bar-Shalom, X. Rong Li, and T. Kirubarajan, Estimation with Applications to Tracking and Navigation. Wiley-Interscience, 2001.

[8] P. Batista, C. Silvestre, and P. Oliveira, "Kalman and $\mathcal{H}_{\infty}$ Optimal Filtering for a Class of Kinematic Systems," in Proc. 17th IFAC World Congress, Seoul, Korea, Jul. 2008.

[9] W. Sun, K. Nagpal, and P. Khargonekar, "H $\mathcal{H}_{\infty}$ Control and Filtering for Sampled-Data Systems," IEEE Trans. on Automatic Control, vol. 38 , no. 8, pp. $1162-1175$, Aug. 1993 .

[10] D. Cabecinhas, C. Silvestre, P. Rosa, and R. Cunha, "Path-Following Control for Coordinated Turn Aircraft Maneuvers," in Proc. AIAA Guidance, Navigation and Control Conf. 2007, Hilton Head, S.C., USA, Aug. 2007. 